\title{
A Case of Systemic Lupus Erythematosus with Cutaneous Leukocytoclastic Vasculitis Mimicking Bullous SLE
}

\author{
Fumi Miyagawa $^{a}$ Kohei Ogawa $^{a}$ Takashi Hashimoto $^{\mathrm{b}}$ Hideo Asada $^{\mathrm{a}}$ \\ aDepartment of Dermatology, Nara Medical University School of Medicine, Kashihara, \\ Japan; ' Department of Dermatology, Osaka City University Graduate School of Medicine, \\ Osaka, Japan
}

\section{Keywords}

Systemic lupus erythematosus · Leukocytoclastic vasculitis · Bullous eruption · Bullous systemic lupus erythematosus

\begin{abstract}
Rarely, patients with systemic lupus erythematosus (SLE) develop bullous eruptions, a disease called bullous SLE in a narrow sense that has autoantibodies against type VII collagen. We describe an unusual case in which a patient with SLE developed extensive bullae on her lower extremities. Histologically, the bullous lesions were suggestive of leukocytoclastic vasculitis with deposition of C3 within blood vessel walls. Immunoblot analyses and enzyme-linked immunosorbent assays were negative for anti-type VII collagen antibodies. We initially considered bullous SLE, but eventually made a diagnosis of secondary vasculitis in SLE. The oral prednisolone dose was increased, and the vesiculobullous lesions resolved. The clinical presentations of cutaneous vasculitis in SLE include palpable purpura, petechiae, papulonodular lesions, and livedo reticularis. Bullous lesions seem to be uncommon. Physicians need to be aware that extensive bullae can occur as a result of secondary vasculitis in SLE, even if the patient does not exhibit high disease activity.
\end{abstract}




\section{Introduction}

It is well known that vasculitis can occur in systemic lupus erythematosus (SLE) patients, but very few studies have specifically examined vasculitis in SLE [1-3]. Thus, there is not much information about the clinical manifestations of vasculitis in SLE. Vasculitis presents in different clinical forms, depending on the size of the affected blood vessels and the organs involved $[4,5]$. Cutaneous lesions, which can manifest as palpable purpura, urticaria, petechiae, papulonodular lesions, livedo reticularis, cutaneous infarctions, erythematous plaques, erythema with necrosis, panniculitis, splinter hemorrhages, and/or superficial ulceration, are the main clinical symptoms $[1,2]$. Bullous lesions seem to be uncommon. Here, we describe a rare case of SLE with secondary vasculitis, in which the patient developed extensive bullous skin lesions on her lower extremities, and we considered bullous SLE and contact dermatitis as potential diagnoses. The absence of antibodies against type VII collagen ruled out bullous SLE in a narrow sense. Extensive bullous lesions caused by secondary vasculitis have rarely been reported in a patient with SLE.

\section{Case Report}

A 33-year-old female had been diagnosed with SLE 10 years ago, when she had developed a fever, a skin rash, arthralgia, anemia, and positivity for anti-dsDNA antibodies ( $27 \mathrm{U} / \mathrm{mL})$. She was followed up by an internist while being treated with $10 \mathrm{mg} /$ day oral prednisolone, $6 \mathrm{mg} /$ day methotrexate, and $3 \mathrm{mg} /$ day tacrolimus for several years. As the arthralgia recurred, treatment with $200 \mathrm{mg}$ /day hydroxychloroquine was added. One month later, she developed a skin rash on her right lower extremity, and the hydroxychloroquine treatment was discontinued. However, the skin rash spread further, so she was referred to our department 5 days later. On examination, she had multiple vesiculobullous eruptions and erythematous macules on her lower extremities (Fig. 1a-c). She also developed erythematous macules on her upper arms (Fig. 1d). Her laboratory data, including her complete blood count, liver and renal function, C-reactive protein, complement component 3 (C3) and C4 levels, $\mathrm{CH} 50$ test results, and erythrocyte sedimentation rate, were all within the normal range. Tests for the following antibodies produced negative results: anti-nuclear, anti-ss DNA, anti-dsDNA, anti-RNP, anti-Sm, anti-SSA/Ro, anti-SSB/La, and anti-cardiolipin antibodies. Tests for PR3-ANCA and MPO-ANCA also produced negative results. Histopathologically, a biopsy from a vesicular skin lesion on her right lower extremity demonstrated edematous changes and superficial perivascular inflammatory infiltrates, consisting of neutrophils and lymphocytes, in the upper dermis, as well as fibrinoid blood vessel necrosis with nuclear debris and extravasated erythrocytes (Fig. 2a, b). The erythema on the upper arms demonstrated similar histological findings, although it did not exhibit edematous changes. Direct immunofluorescence of skin samples from both lesions showed the deposition of C3 (Fig. 3a) and fibrinogen within blood vessel walls. Indirect immunofluorescence of both normal skin and $1 \mathrm{M} \mathrm{NaCl}$-split skin showed negative results. Immunoblot analyses of normal human epidermal extracts, recombinant bullous pemphigoid (BP)180 NC16a and BP180 C-terminal domain proteins, concentrated culture supernatant of HaCaT cells, normal human dermal extracts (Fig. 3b), and purified human laminin-332 all showed negative results. In addition, ELISA was negative for desmoglein 1, desmoglein 3, BP180, BP230, and type VII collagen. A diagnosis of leukocytoclastic vasculitis secondary to SLE was made. The prednisolone dose was increased to $25 \mathrm{mg} /$ day, and the vesiculobullous lesions and erythema resolved. The prednisolone dose was then tapered and maintained at $10 \mathrm{mg} /$ day for 1 year. No recurrence has since been observed.

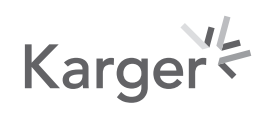




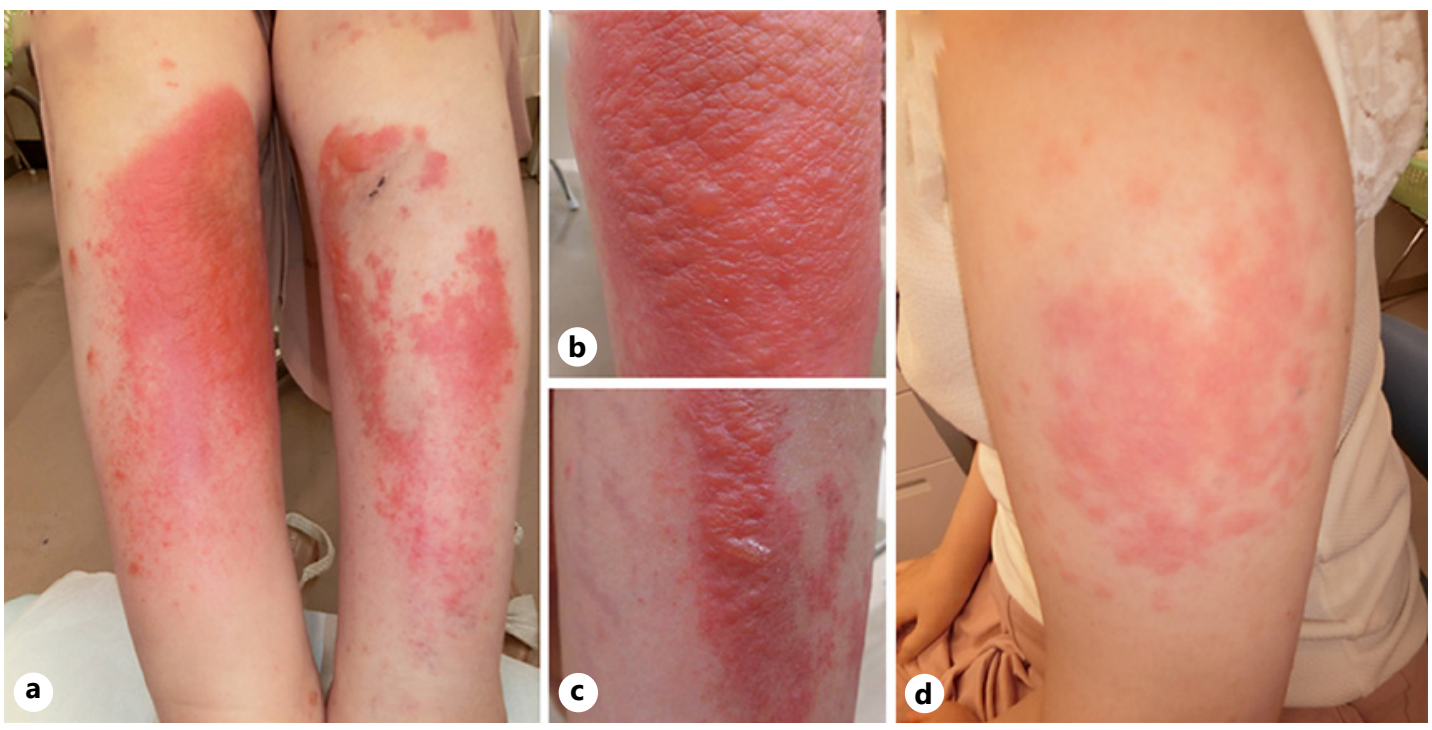

Fig. 1. a Vesiculobullous lesions and erythematous macules on the lower legs. Close-up views of the right lower leg (b) and left lower leg (c). d Erythematous macules on the left upper arm.

\section{Discussion}

Vasculitis is an uncommon, but serious, manifestation of SLE [5]. The prevalence of vasculitis in SLE is reported to range from 11\% [2] to 36\% [1] (visceral and/or cutaneous), while the prevalence of cutaneous vasculitis was reported to range from $10 \%$ [2] to $17.3 \%$ [3]. Episodes of vasculitis are known to often occur during lupus flare-ups accompanied by symptoms, such as fever, fatigue, and weight loss [4]. In addition, a previous study demonstrated that compared with SLE patients without vasculitis, patients with vasculitis had a higher mean European Consensus Lupus Activity Measurement (ECLAM) score (5.86 vs. 3.87; $p<0.001$ ) [2]. However, it is worth noting that episodes of vasculitis do not always occur during periods of high disease activity [4], as was seen in our case.

In 2 large cohort studies of SLE patients with vasculitis, the most common type of vasculitis was small vessel vasculitis (as was seen in our case), which is a type of leukocytoclastic vasculitis that can be limited to the skin $[1,2]$. The clinical presentation of vasculitis in SLE is heterogeneous: the most common skin manifestation is erythematous punctate lesions on the fingertips and palms (36\%), followed by palpable purpura (25\%), ischemic lesions and/or ulcers (14\%), erythematous papules/macules (14\%), urticarial lesions (11\%), and nodular lesions (5\%). Bullous lesions, however, seem to be extremely uncommon. To the best of our knowledge, only 1 case of SLE in which the patient developed severe widespread bullae with histological evidence of leukocytoclastic vasculitis has been reported [6]. However, in the latter case, the cutaneous bullae arose after acute steroid withdrawal, which is different from our case [6].

Up to $29 \%$ of patients develop a combination of the skin manifestations of vasculitis [2]. Our patient also had 2 different types of lesions: erythematous macules on her upper arms and vesiculobullous lesions on her lower legs.

The differential diagnoses in our case included bullous SLE, a blistering disorder that occurs in patients with active SLE, which is characterized clinically by widespread vesiculobullous skin lesions, histopathologically by dermal-epidermal separation in the basement 


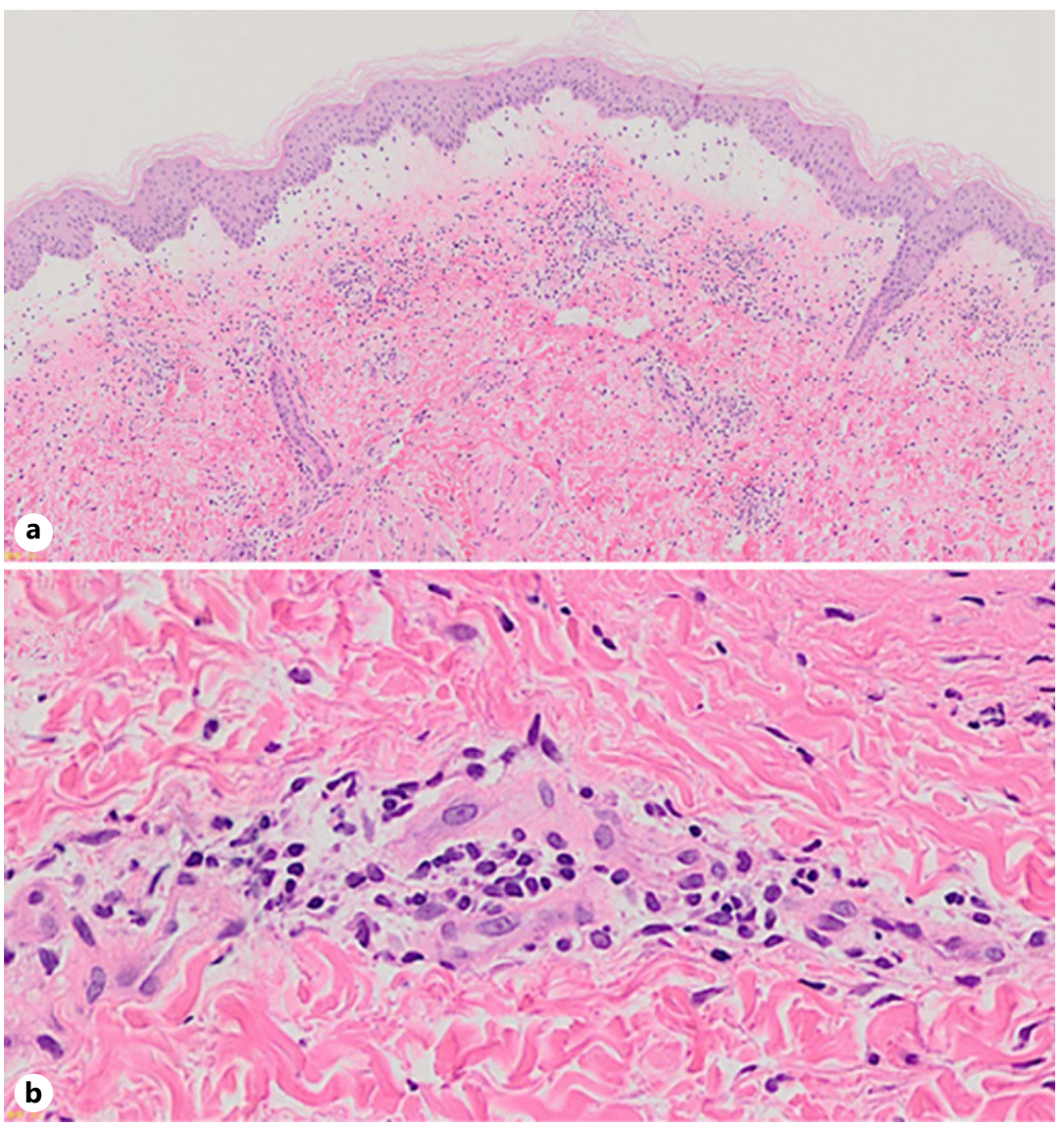

Fig. 2. a A skin biopsy taken from a vesicular lesion on the right lower extremity. Edematous changes and a superficial perivascular inflammatory infiltrate were seen in the upper dermis (hematoxylin and eosin. $\times 40$ ). b Higher magnification $(\times 400)$. A superficial perivascular inflammatory infiltrate, which consisted of neutrophils and lymphocytes, nuclear debris, extravasated erythrocytes, and fibrinoid blood vessel necrosis was seen in the upper dermis.

membrane zone, and immunologically by immunoglobulin deposition in the basement membrane zone in a linear, granular, or mixed linear and granular pattern (shown by direct immunofluorescence) [7]. In bullous SLE, the upper dermis is characterized by edema and the accumulation of neutrophils in dermal papillae, which are similar to the findings seen in dermatitis herpetiformis. In addition, patients with bullous SLE in a narrow sense have autoantibodies to type VII collagen. Another type of bullous lesion that can arise in SLE is epidermal elevation due to vacuolar degeneration of the basal layer and dermal edema [8]. We were able to rule out bullous SLE and contact dermatitis based on the histopathological, immunofluorescence, immunoblotting, and ELISA findings.

As the clinical presentations of the cutaneous lesions of SLE vary significantly, it is sometimes difficult to determine whether cutaneous lesions are related to SLE and/or high SLE disease activity. Physicians need to be aware that extensive bullae can occur as a result of secondary vasculitis in SLE, even if the patient does not exhibit high disease activity. However, since previous studies of vasculitis in SLE were largely based on the clinical manifestations of the condition [3] with only small numbers of vasculitis cases being confirmed by histopathology [1,2], clinical symptoms like those seen in our case might be underreported. 


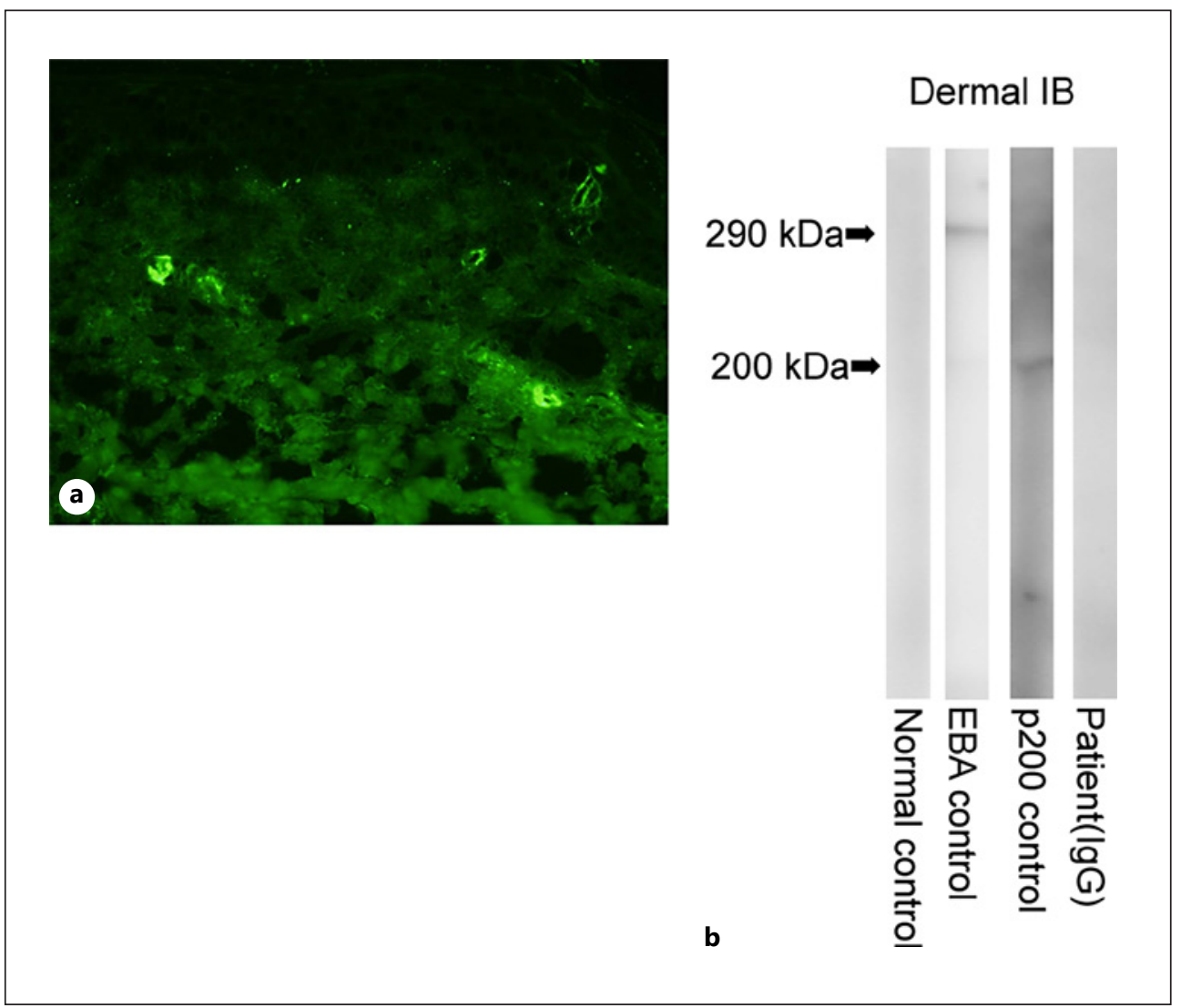

Fig. 3. a Direct immunofluorescence findings. Direct immunofluorescence showed C3 deposition within blood vessel walls. b Immunoblot analysis. Immunoblot analysis of the normal human dermal extract revealed that while the EBA and anti-laminin-gamma 1 (p200) pemphigoid control sera reacted with 290kDa type VII collagen (lane 2) and 200-kDa laminin-gamma 1 (lane 3), the sera from the normal control (lane 1) and our patient (lane 4) produced negative results for these molecules. EBA, epidermolysis bullosa acquisita.

\section{Statement of Ethics}

This research complies with the guidelines for human studies and was conducted ethically in accordance with the World Medical Association Declaration of Helsinki. Written informed consent was obtained from the patient for publication of the details of her medical case and any accompanying images.

\section{Conflict of Interest Statement}

The authors have no conflicts of interest to declare.

\section{Funding Sources}

The authors received no funding for this work. 
Miyagawa et al.: Lupus Vasculitis with Vesiculobullous Eruptions

\section{Author Contributions}

F.M. conceived of and designed the study, analyzed and interpreted the data, and wrote the manuscript. K.O. was responsible for the data analysis and interpretation. T.H. contributed to the data acquisition, analysis, and interpretation and wrote the manuscript. H.A. was responsible for the data interpretation and approved the manuscript.

\section{Data Availability Statement}

All data generated or analyzed during this study are included in this article. Further enquiries can be directed to the corresponding author.

\section{References}

1 Drenkard C, Villa AR, Reyes E, Abello M, Alarcón-Segovia D. Vasculitis in systemic lupus erythematosus. Lupus. 1997;6:235-42.

2 Ramos-Casals M, Nardi N, Lagrutta M, Brito-Zerón P, Bové A, Delgado G, et al. Vasculitis in systemic lupus erythematosus: prevalence and clinical characteristics in 670 patients. Medicine. 2006;85:95-104.

3 Kallas R, Goldman D, Petri MA. Cutaneous vasculitis in SLE. Lupus Sci Med. 2020;7:e000411.

4 Barile-Fabris L, Hernández-Cabrera MF, Barragan-Garfias JA. Vasculitis in systemic lupus erythematosus. Curr Rheumatol Rep. 2014;16(9):440.

5 Calle-Botero E, Abril A. Lupus vasculitis. Curr Rheumatol Rep. 2020;22:71.

6 Callen JP. Cutaneous bullae following acute steroid withdrawal in systemic lupus erythematosus. Br J Dermatol. 1981;105:603-6.

7 Gammon WR, Briggaman RA. Bullous SLE: a phenotypically distinctive but immunologically heterogeneous bullous disorder. J Invest Dermatol. 1993;100:28S-34S

8 Camisa C, Sharma HM. Vesiculobullous systemic lupus erythematosus. Report of two cases and a review of the literature. J Am Acad Dermatol. 1983;9:924-33. 